\title{
COMMUNICATION
}

\section{SYNTHESIS AND X-RAY CRYSTAL STRUCTURE OF A DINUCLEAR PLATINUM HYDRIDE COMPLEX OF TETRACYANOBIIMIDAZOLE}

\author{
J. CARLOS BAYÓN \\ Departament de Química, Universitat Autònoma de Barcelona, Bellaterra, Barcelona, \\ Spain \\ and

\section{J. BRUCE KOLOWICH and PAUL G. RASMUSSEN*}

Department of Chemistry, University of Michigan, Ann Arbor, MI 48109, U.S.A.

(Received 19 June 1986; accepted 15 August 1986)

\begin{abstract}
Reaction of the diacid form of tetracyanobiimidazole via oxidative addition with two equivalents of $\mathrm{Pt}\left(\mathrm{PPh}_{3}\right)_{4}$ produces a dinuclear complex whose structure is solved by $\mathrm{X}$-ray analysis. The presence of dihydride is confirmed by observation of IR shift of a deuterium-labelled compound.
\end{abstract}

The reaction of protic acids with platinum(0)tetrakisphosphines via oxidative addition to form platinic hydrides has been well documented in the literature. Earlier works have described numerous examples of reaction of $\mathrm{Pt}(0)$ with mineral acids ${ }^{1}$ and imides ${ }^{2}$ to produce such hydride compounds, but reactions with oxyacids do not produce compounds of similar stability. ${ }^{3}$ Hydride-containing dinuclear complexes of platinum without bridging hydrides are rare, ${ }^{4-7}$ and are not the product of reaction of a diacid with two equivalents of a $\mathrm{Pt}(0)$ species.

We now report the synthesis of a symmetrical

*Author to whom correspondence should be addressed

†Crystal data: $\mathrm{C}_{82} \mathrm{H}_{62} \mathrm{~N}_{8} \mathrm{P}_{2} \mathrm{Pt}_{2}, M=1611.58$, triclinic, space group $P \bar{I}, a=13.397 \AA, b=14.734 \AA, c=12.379 \AA$, $\alpha=100.82^{\circ}, \beta=115.90^{\circ}, \gamma=64.65^{\circ}, U=1986 \AA^{3}, Z=1$, $D_{\text {calc }}=1.408 \mathrm{~g} \mathrm{~cm}^{-3}, \mu\left(\mathrm{Mo}-K_{a}\right)=36.81 \mathrm{~cm}^{-1}, \lambda(\mathrm{Mo}-$ $\left.K_{a}\right)=0.71069 \AA, N=4614, N P=479, R=0.0283$, nonhydrogen atoms anisotropic, hydrogens fixed but not refined. Tables of atomic positional and thermal parameters, bond lengths and angles and $F_{\mathrm{o}} / F_{\mathrm{c}}$ values have been deposited as supplementary material with the Editor from whom copies are available on request. Atomic coordinates have also been deposited with the Cambridge Crystallographic Data Centre.
platinum(II)hydrido dinuclear compound formed by the oxidative addition of a non-oxygen diacid, $4,4^{\prime}, 5,5^{\prime}$-tetracyano-2,2'-biimidazole $\left(\mathrm{C}_{10} \mathrm{~N}_{8} \mathrm{H}_{2}\right)$, to two equivalents of tetrakistriphenylphosphineplatinum( $(0)$. The white, air-stable reaction product, $\mathrm{HPt}\left(\mathrm{PPh}_{3}\right)_{2}\left(\mathrm{C}_{10} \mathrm{~N}_{8}\right)\left(\mathrm{PPh}_{3}\right)_{2} \mathrm{PtH}$, precipitates quantitatively on stirring for $1 \mathrm{~h}$ in tetrahydrofuran, regardless of the stoichiometric ratios added to the reaction mixture. Crystals of this compound suitable for X-ray analysis were prepared by allowing a mixture of the reactants to stand overnight in a new flask. Once the compound was prepared, it showed no solubility in common laboratory solvents except those which react with the hydride.

Chemical characterization of the dinuclear compound included elemental analysis and IR spectra. Principle characterization of the structure was the result of X-ray analysis (Fig. 1). $\dagger$ From this we find the geometry about the platinum to be a slightly distorted square-planar, with angles $\mathrm{P} 1-\mathrm{Pt}-\mathrm{N} 1$ $99.8^{\circ}$ and $\mathrm{P} 2-\mathrm{Pt}-\mathrm{N} 195.0^{\circ}$. The atoms $\mathrm{P} 1, \mathrm{P} 2$, $\mathrm{Pt}$ and $\mathrm{N} 1$ are coplanar, and this plane is almost exactly perpendicular to that of the central ligand. The bonded distance of $2.125 \AA$ for $\mathrm{Pt}-\mathrm{N} 1$ compares to the non-bonded distance of $3.027 \AA$ for $\mathrm{Pt}-\mathrm{N} 3^{\prime}$. Examination of packing plots from all axes revealed no strong intermolecular association. 


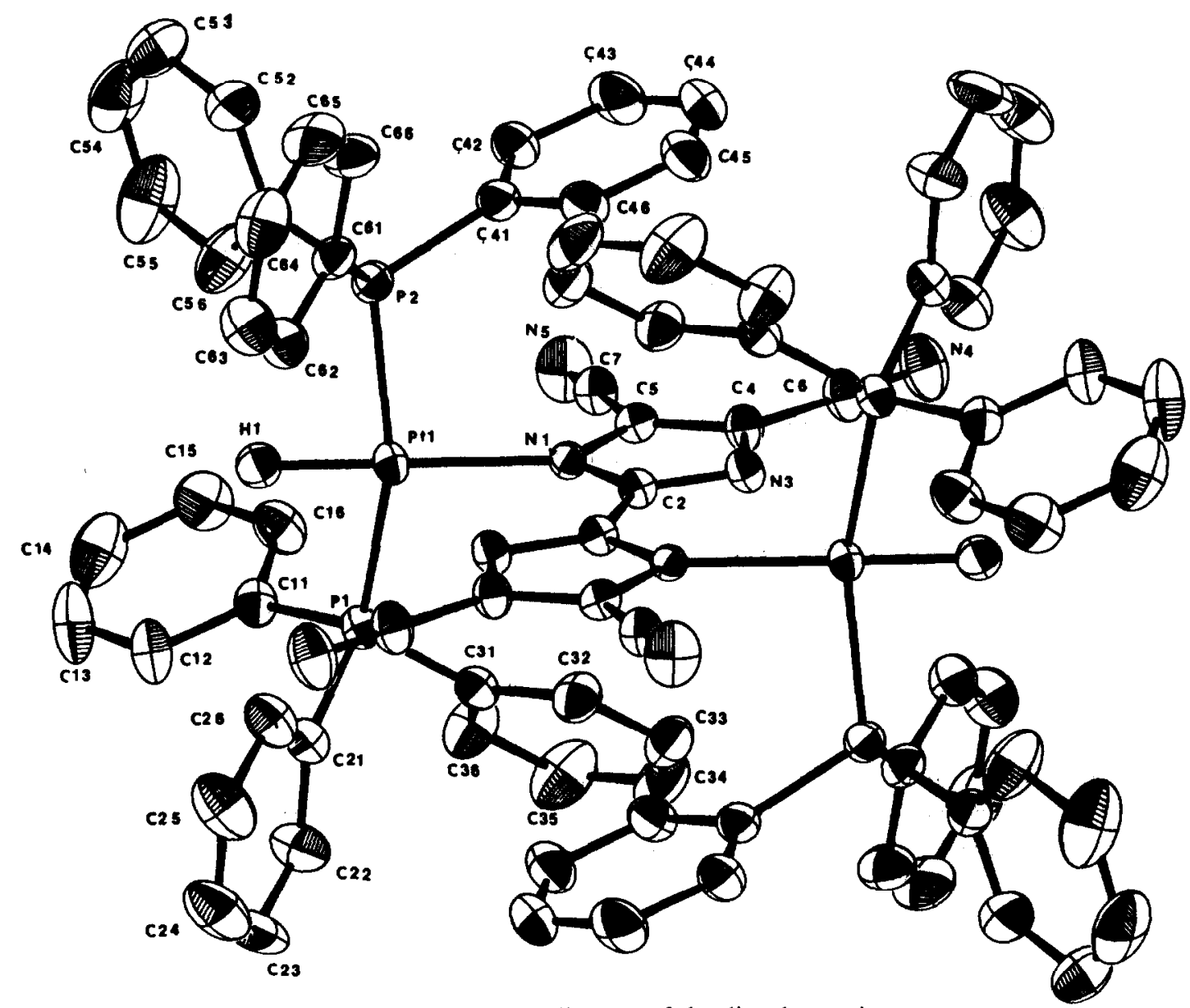

Fig. 1. ORTEP diagram of the dinuclear unit.

Stereoplots of these packings indicated that interaction was mainly confined to the phenyl ring periphery of the molecule. This is the first example of coordination of this type for tetracyanobiimidazole. More commonly expected are dinuclear species with quadridentate binding to the ring nitrogens, ${ }^{8}$ or mononuclear bidentate species with the metal atom bound to one side of the ligand.

The hydride was verified via isotopic labeling and observance of the shift in frequency for the IR $\mathrm{Pt}-\mathrm{H}$ resonance. Labeling was accomplished by stirring the acid form of the ligand in $\mathrm{D}_{2} \mathrm{O}$ at $5^{\circ} \mathrm{C}$ for several hours. This deuterated form of the ligand $\left(D_{2} C_{10} N_{8}\right)$ was then allowed to react with two equivalents of tetrakistriphenylphosphineplatinum(0). The resulting product was identical in appearance and solubility to the protic analog. IR spectra of the two compounds were virtually identical, except for the hydride-deuteride bands. The hydride band at $2196 \mathrm{~cm}^{-1}$ was absent in the deuteride spectrum, and as expected, a new resonance had appeared at $1621 \mathrm{~cm}^{-1}$.

Investigations of this unusual molecule are con- tinuing with synthetic attempts to probe the hydride reactivity.

Acknowledgements-P.G.R. acknowledges support from the donors of the Petroleum Research Fund administered by the American Chemical Society. J.C.B. acknowledges support from CIRIT (Catalonia). J.B.K. acknowledges support from the H. Judson Osterhof fellowship of the Rackham Graduate School.

\section{REFERENCES}

1. F. Cariati, R. Ugo and F. Bonati, Inorg. Chem. 1966 , 5, 1128.

2. D. M. Roundhill, Inorg. Chem. 1970, 9, 254.

3. D. Carmona, R. Thouvenot, L. M. Venanzi, F. Bachechi and L. Zambonelli, J. Organomet. Chem. 1983, 250, 589.

4. R. S. Paonessa, A. L. Prignano and W. C. Trogler, Organometallics 1985, 4, 647.

5. R. S. Paonessa and W. C. Trogler, Inorg. Chem. 1983, 22, 1038.

6. M. Auburn, M. Ciriano, J. A. K. Howard, M. Murray, N. J. Pugh, J. L. Spencer, F. G. A. Stone and P. 
Woodward, J. Chem. Soc., Dalton Trans. 1980, 659.

7. T. H. Tulip, T. Yamagata, T. Yoshida, R. D. Wilson, J. A. Ibers and S. Otsuka, Inorg. Chem. 1979, 18, 2239.

8. P. G. Rasmussen, J. E. Anderson and J. C. Bayón, Inorg. Chim. Acta 1984, 87, 159.

9. P. G. Rasmussen, J. E. Anderson, O. H. Bailey and J. C. Bayón, J. Am. Chem. Soc. 1985, 107, 279.
Woodward, J. Chem. Soc., Dalton Trans. 1980, 659. 7. T. H. Tulip, T. Yamagata, T. Yoshida, R. D. Wilson, J. A. Ibers and S. Otsuka, Inorg. Chem. 1979, 18, 2239. 8. P. G. Rasmussen, J. E. Anderson and J. C. Bayón, Inorg. Chim. Acta 1984, 87, 159.

9. P. G. Rasmussen, J. E. Anderson, O. H. Bailey and J. C. Bayón, J. Am. Chem. Soc. 1985, 107, 279. 